\title{
Perceptions of disadvantage, ethnicity and psychosis
}

C. Cooper, C. Morgan, M. Byrne, P. Dazzan, K. Morgan, G. Hutchinson, G. A. Doody,

G. Harrison, J. Leff, P. Jones, K. Ismail, R. Murray, P. E. Bebbington and P. Fearon

\section{Background}

People from Black ethnic groups (African-Caribbean and Black African) are more prone to develop psychosis in Western countries. This excess might be explained by perceptions of disadvantage.

\section{Aims}

To investigate whether the higher incidence of psychosis in Black people is mediated by perceptions of disadvantage.

\section{Method}

A population-based incidence and case-control study of firstepisode psychosis (Aetiology and Ethnicity in Schizophrenia and Other Psychoses (AESOP)). A total of 482 participants answered questions about perceived disadvantage.

\section{Results}

Black ethnic groups had a higher incidence of psychosis
(OR=4.7, 95\% Cl 3.1-7.2). After controlling for religious affiliation, social class and unemployment, the association of ethnicity with psychosis was attenuated $(\mathrm{OR}=3.0,95 \% \mathrm{Cl} 1.6-5.4)$ by perceptions of disadvantage. Participants in the Black non-psychosis group often attributed their disadvantage to racism, whereas Black people in the psychosis group attributed it to their own situation.

\section{Conclusions}

Perceived disadvantage is partly associated with the excess of psychosis among Black people living in the UK. This may have implications for primary prevention.

\section{Declaration of interest}

None.
The Aetiology and Ethnicity in Schizophrenia and Other Psychoses (ÆSOP) study has confirmed that the incidence of psychosis is significantly higher in African-Caribbean and Black African groups living in the UK than in the White British population. ${ }^{1}$ Sharpley et al identified putative explanations for this, including a psychological hypothesis that interpretation of experiences as discriminatory might account for these increased rates, and a social hypothesis that greater objective social disadvantage and racism might increase the likelihood of experiencing psychosis. ${ }^{2}$ There is some support for both of these hypotheses in the literature. Gilvarry et al reported that people with severe mental illness from Black and minority ethnic groups were more likely than their White counterparts to attribute life events to discrimination. ${ }^{3}$ Karlsen \& Nazroo found that among participants of Caribbean, Irish, Bangladeshi, Pakistani and Indian ethnicities in the Ethnic Minority Psychiatric Illness Rates in the Community (EMPIRIC) study, the prevalence of psychosis was three times higher in those who reported experiencing verbal racism, and five times higher in those reporting a racist physical attack, than in those who did not, ${ }^{4}$ supporting the social hypothesis above.

Direct racist attack is less common than perceived discrimination in interpersonal interactions. ${ }^{5}$ Using data from a large, crosssectional national survey in Sweden, Wamala et al demonstrated that perceived discrimination, defined as unfair treatment that results in humiliation, was associated with psychological distress. ${ }^{6}$ Veling et al demonstrated that the rates of self-reported discrimination among people living in a Dutch city were higher among people from non-Western ethnic groups (mainly people originating from Surinam, Turkey and Morocco) compared with those from Western ethnic groups, and that this partially explained the greater likelihood of psychosis in the non-Western ethnic groups. $^{7}$

In this study we distinguish between the perception of disadvantage and its attribution (for instance, to discrimination, to mental illness or to personal failings). We primarily aimed to use data from the ÆSOP study in order to test the hypothesis that the increased incidence of psychosis among Black people living in the UK is mediated by higher rates of perceived disadvantage. We also explored participants' own accounts of the reasons for perceived disadvantage, including whether they attributed this to mental illness. Our second hypothesis was that reporting higher levels of disadvantage would be more common in people with psychosis after taking account of ethnic status and other socioeconomic factors. Finally, we investigated whether there was an association in people with psychosis between perceived disadvantage and (first) self-esteem and self-concept, and (second) psychotic symptoms. The purpose of this was to explore whether the association between psychosis and perceived disadvantage might be accounted for by low self-esteem and poor self-concept or by psychotic symptoms such as delusions of persecution or reference.

\section{Method}

\section{Recruitment}

The ÆSOP study is a population-based incidence and casecontrol study of first-episode psychosis. The inclusion criteria for cases were age 16-65 years; residence within tightly defined catchment areas in Nottingham or south-east London; presence of a first episode of psychosis (diagnostic codes F10-F29 and F30-F33 in ICD-10) ${ }^{8}$ within the time frame of the study; and no previous contact with health services for psychosis. The initial study also included a centre in Bristol, but this was omitted from the study reported here as only six of the participants from that centre had completed the measure of perceived disadvantage. Exclusion criteria were evidence that psychotic symptoms had an organic cause, and transient psychotic symptoms resulting from acute intoxication, as defined by ICD-10. No patient included in the study had an IQ of less than 65. Recruitment of 
all participants took place between 1997 and 2003. A team of researchers regularly checked all points of potential patient contact with secondary and tertiary health services in the catchment areas. All potential cases were screened for inclusion using the Screening Schedule for Psychosis. ${ }^{1}$

A random population-based sample of control participants was selected using the sampling frame preferred by the Office of Population and Census Statistics Psychiatric Morbidity Survey, namely the Postal Address File. ${ }^{9}$ The Postal Address File was used to generate a random sample of ten target addresses in relation to each case, from which controls were recruited. Each address was contacted three times (morning, afternoon, evening) to find eligible and willing control participants aged $16-65$ years. This method matches cases and controls by area of residence. Cases and controls were otherwise unmatched, the aim being to select a sample of controls representative of the population from which the cases were drawn. Candidates for the control group were excluded if they screened positive for psychosis on assessment with the Psychosis Screening Questionnaire. ${ }^{10}$ African-Caribbean controls were oversampled to ensure sufficient numbers.

During the study period 535 cases (330 in south-east London, 205 in Nottingham) and 391 controls (183 in south-east London, 208 in Nottingham) were identified, a total of 926 participants; the response rate for the main study was $66 \%$ (see earlier ÆSOP papers for more details of the study). ${ }^{11,12}$ Five hundred and seventeen participants $-41.9 \%(n=224)$ of cases and $74.9 \%(n=293)$ of controls - completed the questions on disadvantage. Of these, we included those from the Black (Black Caribbean $n=108$, Black African $n=32$, Black other $n=2$; total $n=142$ ) and White (British $n=305$, Irish or other $n=35$; total $n=340$ ) ethnic groups in the current study.

\section{Measures}

Data on ethnicity, gender, age and social class were collected using the Medical Research Council Socio-demographic Schedule. ${ }^{13}$ Ethnicity was based on participant self-ascription using 2001 census categories. Social class was based on the participants' highest-level occupation in 1990, or their father's last occupation if they had never worked. We also extracted data on the highest educational level attained (school, further, higher); whether participants were born in the UK or not; employment status (coded as employed, unemployed, other); religion (coded as Christian, Muslim, other and none); and recruitment centre (London or Nottingham).

The Culture and Identity Schedule-2 (CANDID-2) questionnaire, developed from an earlier instrument, ${ }^{14}$ explores the attitudes and perceptions of participants to life in the UK today. As part of this questionnaire participants were asked, 'Do you believe that you experience any disadvantage when compared with other individuals in British society?' and requested to rate their answers on a Likert scale from 1 (no), 3 (partly) to 5 (yes). Those rating their answers $2-5$ were then asked to rate, also on five-point Likert scales, the degree to which they thought this was due to their skin colour; to their culture, cultural beliefs or religion; or to their social class, and invited to make additional comments about possible reasons. They were asked what else might be responsible for the disadvantage that they experienced. Answers were recorded verbatim, and subsequently classified by one of the authors (C.C.) according to the type of reason given. Finally, respondents were asked, 'What do you think determines your social class?' Interviewers then probed for the responses: family background, education, race, address, culture, clothing, accent, wealth or income. All determinants listed by the participants were recorded.
Patients were interviewed using the World Health Organization (WHO) Schedules for Clinical Assessment in Neuropsychiatry (SCAN) version 2.1. ${ }^{15}$ A total symptom score was obtained by summing the SCAN's individual symptom item scores, as per Wing \& Sturt's procedure for the Present State Examination. ${ }^{16}$ Scores for persecutory delusions, delusions of reference and other non-affective delusions, and for auditory hallucinations were analysed as below threshold (score 0), present and associated with moderate disability (score 1) or present with severe disability or distress (score 2). Participants also completed the Rosenberg Self-Esteem Scale, ${ }^{17,18}$ a ten-item questionnaire with items rated on a four-point Likert scale which assesses respondents' overall evaluation of themselves, and the Robson self-concept questionnaire. ${ }^{19}$

\section{Data analysis}

In all analyses we weighted data (using the pweight command in Stata 8.0 for Windows) to account for the oversampling of African-Caribbean controls, using weights of 0.48 and 0.18 respectively for African-Caribbean controls from London and Nottingham. We compared the socio-demographic characteristics of those with psychosis and those without who had completed the CANDID-2 questionnaire. We then conducted a series of logistic regressions with case status (psychosis case or control) as the dependent variable. To test our first hypothesis, that perceived disadvantage mediates the association between ethnicity and case status, we used the following steps: first, we determined whether ethnicity (the main explanatory variable) was associated with perceived disadvantage; second, we determined whether ethnicity was associated with psychosis (outcome) using logistic regression adjusting for age, centre and gender, and reporting as odds ratio; third, the odds ratio for relationship of perceived disadvantage (mediator) with psychosis was determined; fourth, assuming that the first three steps confirm a positive association, we added perceived disadvantage to the logistic regression in step 2, to determine whether the size of the association between ethnicity and psychosis was reduced. ${ }^{20}$

We determined whether reports of perceived disadvantage correlated with any of the psychosis symptoms (in the case group), self-esteem and self-concept scores (in the whole sample), using Spearman's rho correlations, to explore whether reports of disadvantage might plausibly be related to specific symptoms. We repeated the logistic regression above with self-esteem and selfconcept as the added independent variables, to determine whether they attenuated the relationship between ethnicity and case status. To test our second hypothesis that people with psychosis experienced more disadvantage after controlling for socio-economic variables, we entered all the socio-economic variables studied that approached significance $(P<0.1)$ on univariate analysis in an overall model.

We investigated whether the categories (skin colour, culture and social class) of disadvantage were more likely to be experienced by people with psychosis compared with controls after controlling for ethnicity, age and gender. Finally, we qualitatively described and summarised the factors cited as contributing to social class, and additional reasons given for experiencing disadvantage in those with psychosis and those without.

\section{Results}

People with psychosis were younger, and more likely to be unemployed, to belong to a Black ethnic group, to have left school at age 18 years or before, to come from social class V (unskilled) and to claim adherence to some form of religion (Table 1). They were 


\begin{tabular}{|c|c|c|c|c|}
\hline & Cases group & Control group & OR $(95 \% \mathrm{Cl})$ & $P$ \\
\hline Age, years: mean (s.e.) & $32.1(0.84)$ & $38.7(0.79)$ & $0.96(0.94-0.97)$ & $<0.001$ \\
\hline Male gender, $n(\%)$ & $102(51.8)$ & $122(42.8)$ & $0.70(0.48-1.0)$ & 0.057 \\
\hline \multicolumn{5}{|l|}{ Ethnicity, $n(\%)$} \\
\hline White British & $118(59.9)$ & $222(87.6)$ & $4.7(3.1-7.2)$ & $<0.001$ \\
\hline Black & $79(40.1)$ & $63(12.4)$ & & \\
\hline \multicolumn{5}{|l|}{ Employment status, $n$ (\%) } \\
\hline Unemployed & $115(58.4)$ & $116(40.7)$ & 1.0 & \\
\hline Employed & $61(31.0)$ & $156(54.7)$ & $0.38(0.26-0.57)$ & $<0.001$ \\
\hline Other & $21(10.7)$ & $13(4.6)$ & $1.7(0.79-3.6)$ & 0.17 \\
\hline \multicolumn{5}{|l|}{ Level of education, $n$ (\%) } \\
\hline School & $117(59.4)$ & $135(47.7)$ & 1.0 & \\
\hline Further & $55(27.9)$ & $86(30.4)$ & $0.74(0.48-1.1)$ & 0.18 \\
\hline Higher & $25(12.7)$ & $62(21.9)$ & $0.44(0.26-0.74)$ & 0.002 \\
\hline \multicolumn{5}{|c|}{ Participant's social class, ${ }^{a}$ n (\%) } \\
\hline Professional & $6(3.4)$ & $12(4.5)$ & 1.0 & \\
\hline Intermediate & $19(10.9)$ & $93(34.7)$ & $0.45(0.15-1.4)$ & 0.16 \\
\hline Skilled non-manual & $45(25.7)$ & $64(23.9)$ & $1.6(0.57-4.7)$ & 0.36 \\
\hline Skilled manual & $37(21.1)$ & $36(13.4)$ & $2.1(0.71-6.3)$ & 0.18 \\
\hline Semi-skilled & $45(25.7)$ & $51(19.0)$ & $2.1(0.72-6.1)$ & 0.17 \\
\hline Unskilled & $23(13.1)$ & $12(4.5)$ & $4.5(1.3-15.4)$ & 0.015 \\
\hline \multicolumn{5}{|l|}{ Religion, n (\%) } \\
\hline Christian & $110(57.3)$ & $130(45.8)$ & 1.0 & \\
\hline Muslim & $4(2.1)$ & $1(0.35)$ & $4.3(0.46-38.9)$ & 0.2 \\
\hline Other & 25 (13.0) & $25(8.8)$ & $1.3(0.70-2.5)$ & 0.40 \\
\hline None & $53(27.6)$ & $128(45.1)$ & $0.49(0.32-0.74)$ & 0.001 \\
\hline UK-born, n (\%) & $157(79.7)$ & $235(82.5)$ & $0.75(0.47-1.2)$ & 0.24 \\
\hline
\end{tabular}

also more likely than the control group to think they were at a greater disadvantage than other people in Britain $(\mathrm{OR}=1.3,95 \%$ CI 1.1-1.5; $P<0.001)$. The self-esteem scores were lower for psychosis cases (mean 35.9, 95\% CI 34.8-37.0) than for controls (mean 139.0, 95\% CI 38.1-40.0). Self-concept scores were also lower for cases (mean 117.0, 95\% CI 111.6-122.4) than for controls (mean 138.3, 95\% CI 134.0-142.5). Overall perceptions of disadvantage scores were higher in Black participants (mean score 3.0, s.e. $=0.15$ ) than in White participants (mean score 1.8, s.e. $=0.17)$. Greater perception of disadvantage correlated significantly with lower self-esteem $(\rho=-0.14, P=0.003)$ and self-concept $(\rho=-0.12, P=0.016)$ scores, but not with scores for persecutory delusions $(\rho=-0.055, P=0.46)$, delusions of reference $(\rho=-0.011, P=0.89)$, non-affective auditory hallucinations $(\rho=-0.021, P=0.78)$ or non-specific auditory hallucinations $(\rho=0.051, P=0.50)$.

After controlling for age and gender, people from Black ethnic groups were over four times more likely to have psychosis than White people (Table 2). The association between Black ethnicity and case status was reduced by controlling for socio-economic factors (social class, educational level, religious adherence, employment) and for greater perception of disadvantage, confirming evidence of mediation, but increased by controlling for self-esteem and self-concept. In our final model (Table 3), being younger, unemployed and from a Black ethnic group, expressing adherence to some form of religion and perceiving more disadvantage were the significant factors predicting case status.

\section{Reasons for perceived disadvantage}

People with psychosis were more likely than those in the control group to report experiencing disadvantage due to skin colour ( $\mathrm{OR}=1.2,95 \%$ CI $1.1-1.4 ; P=0.009)$, but findings for cultural beliefs and religion $(\mathrm{OR}=1.2,95 \% \mathrm{CI} 0.96-1.4 ; P=0.12)$ and social class $(\mathrm{OR}=1.2,95 \% \mathrm{CI} 0.98-1.4 ; P=0.091)$ were not statistically significant. However, once the higher perception of disadvantage scores by Black people, who were more likely to be categorised as cases, were controlled for, people with psychosis were no more likely to report perceiving disadvantage due to cultural beliefs and religion or to social class, and they were actually less likely to report disadvantage due to skin colour $(\mathrm{OR}=0.82,95 \% \mathrm{CI}$ $0.68-0.98 ; P=0.027)$. The proportion of people who reported being disadvantaged due to culture or religion at least part of the time (score 3 or above) was greater in Black people $(26.1 \%)$ than in White people $(6.0 \%)$; this was also true for reports of disadvantage due to skin colour $(55.0 \% v .4 .8 \%)$ and social class (27.1\% v. $12.1 \%)$.

\section{Qualitative analyses}

\section{Culture and religion}

Several respondents mentioned specific situations in which they felt they were disadvantaged because of their culture, most frequently in seeking employment. One person with psychosis originally from Yugoslavia described 'snide remarks from colleagues about taking jobs at the expense of British people'. Several reported tensions emanating from a clash of their own and British culture. One Black person with psychosis commented, 'I was supposed to follow my culture but was prevented from doing this.' Others who made additional comments attributed cultural disadvantage to their physical appearance (e.g. dreadlocks, mode of dress), or language (e.g. accent, disapproval of communication in their own language in public). Some described negative assumptions made by others as a result of their culture. One Black person recruited as a control commented, 'I didn't feel people of my colour could achieve and this seemed to be confirmed by my teachers'. A Black person with psychosis commented, '[The discrimination] boils down to what happens to 
Table 2 Odds ratios for relationship between case status and Black ethnic group, adjusted for factors studied

\begin{tabular}{|c|c|}
\hline & OR $(95 \% \mathrm{Cl})^{\mathrm{a}}$ \\
\hline Not adjusted for further factors & $4.5(2.7-7.4)^{\star * *}$ \\
\hline Adjusted for overall perception of disadvantage & $4.1(2.5-6.8)^{* * *}$ \\
\hline Adjusted for perception of disadvantage due to skin colour & $6.9(3.7-13.0)^{* * *}$ \\
\hline Adjusted for perception of disadvantage due to culture & $5.0(3.0-8.3)^{* * *}$ \\
\hline Adjusted for perception of disadvantage due to social class & $4.6(2.8-7.7)^{\star \star *}$ \\
\hline Adjusted for social class, educational level, employment status and religion & $3.5(2.0-6.2)^{* * *}$ \\
\hline Adjusted for self-esteem and self-concept & $7.6(4.1-14.1)^{* * *}$ \\
\hline
\end{tabular}

us and our history. Whites will never fully respect us.' Another stated that, 'The people in power don't like Black culture'. Three people (all categorised as cases: one from a White group and two from Black groups) specifically commented about racism.

\section{Skin colour}

One person with psychosis commented, 'You're seen as just a Black person'; another that Black men were 'seen as aggressive'. Twelve White people from the control group (compared with one from the cases group) reported feeling that people from ethnic minorities were given preferential treatment in British society.

\section{Social class}

The majority of comments concerned lack of opportunities in education and employment due to being from a lower social class. When asked what determined social class, the most frequently endorsed responses were wealth or income $(n=233 ; 48.3 \%)$, family background $(n=215 ; 48.2 \%)$, education $(n=168 ; 37.7 \%)$, occupation $(n=101 ; 21.0 \%)$, address $(n=58 ; 12.0 \%)$ and the person's own behaviour and attitudes $(n=40 ; 8.3 \%)$. There was no significant difference in the proportion of people with and without psychosis citing these factors.

\section{Other reasons for disadvantage}

Finally, we investigated other reasons given for perceiving disadvantage in those with psychosis $(n=67)$ and those without $(n=64)$. The most frequent answers were gender $(8$ cases, 9 controls); health or disability (physical or unspecified; 5 cases, 9 controls); racism, nationality or asylum status ( 6 cases, 9 controls); lack of education or support at school (10 cases, 3 controls); family background, problems or lack of support ( 8 cases, 3 controls); own lack of confidence, attitude or behaviour ( 7 cases, 2 controls); financial factors ( 2 cases, 7 controls); social class, profession or social exclusion ( 3 cases, 5 controls); age ( 2 cases, 6 controls); the negative attitudes of others ( 4 cases, 4 controls); appearance or height ( 5 cases, 1 control); manner of speech, accent or use of language (1 case, 2 controls) and bad luck (3 cases, 0 controls). Only one respondent (with psychosis) specifically mentioned mental health as a reason for discrimination.

\section{Discussion}

We found that the association between belonging to a Black ethnic group and psychosis was partially mediated by perceived disadvantage, as well as by socio-economic disadvantages. This concurs with findings from the only previous study to investigate perceived disadvantage as a potential mediator of the relationship between risk of psychosis and ethnic group, ${ }^{7}$ and suggests that those results hold true for the excess of psychosis cases reported among the UK Black population. Our second hypothesis, that people with psychosis would report more disadvantage than controls after taking account of socio-economic factors including ethnicity, was also supported.

This research touches on two key theoretical issues in psychosis. The first is how social contexts shape the content of psychotic symptoms. ${ }^{21}$ The second is the central role of appraisal in cognitive models of psychosis. ${ }^{22}$ One might predict that perceiving disadvantage would give rise to paranoid thoughts. It is clear that incidents of discrimination occur, and in any given society there will be groups who experience them more often than others, and ethnic groups for whom the experience is qualitatively different. Where individuals appraise such incidents as personally directed, they may regard them as persecutory, and persecutory patterns of thought may be initiated. If so, this would represent a direct cognitive pathway into paranoid interpretations. The converse, that a paranoid view of the world may increase the perception of experiences as disadvantageous, would also seem a reasonable assumption. It is therefore surprising that we found no correlation between perceived disadvantage and persecutory

Table 3 Logistic regression of factors predicting case

status (psychosis)

\begin{tabular}{|llc|}
\hline Factor & OR $(95 \% \mathrm{Cl})$ & $P$ \\
\hline Age & $0.96(0.95-0.98)$ & $<0.001$ \\
\hline Male gender & $0.78(0.48-1.3)$ & 0.30 \\
\hline Centre (London, reference) & $0.68(0.41-1.1)$ & 0.14 \\
\hline Black ethnicity (White, reference) & $3.0(1.6-5.4)$ & $<0.001$ \\
\hline Religion & & \\
$\quad$ Christian (reference) & 1 & 0.93 \\
Muslim & $1.1(0.15-8.0)$ & 0.58 \\
Other & $0.78(0.32-1.9)$ & $<0.001$ \\
\hline None & $0.38(0.23-0.63)$ & \\
\hline Social class & & 0.063 \\
Professional (reference) & 1 & 0.51 \\
Intermediate & $0.32(0.095-1.1)$ & 0.85 \\
Skilled non-manual & $0.66(0.19-2.3)$ & 0.69 \\
Skilled manual & $1.1(0.33-3.9)$ & 0.41 \\
\hline Semi-skilled & $0.77(0.22-2.8)$ & \\
Unskilled & $1.8(0.46-6.9)$ & 0.020 \\
\hline Employment & & 0.009 \\
$\quad$ Not employed (reference) & 1 & 0.17 \\
\hline Employed & $0.53(0.32-0.85)$ & \\
Student & $2.2(0.72-6.7)$ & \\
\hline Education level & & \\
School (reference) & $1.2(1.0-1.4)$ & \\
Further & & \\
Higher & $0.76(0.43-1.3)$ & \\
\hline Perception of disadvantage & $0.83(0.39-1.8)$ & \\
\hline
\end{tabular}


delusions or other measures of psychotic symptoms in our study. This suggests that the participants' recognition of disadvantage was not greatly distorted by specific psychotic patterns of thinking.

Black people with psychosis reported particularly high rates of disadvantage, but they were no more likely than other Black people to attribute this to their culture, religion, social class or skin colour, and they were less likely than other Black people to include the possibility of racism in their comments. Their greater perception of disadvantage must therefore have other attributions. When asked to comment upon the reasons for their disadvantages, people with psychosis referred more frequently than controls to lack of support at school or home, their own lack of confidence, attitudes, behaviour or physical appearance, and bad luck: only one participant mentioned mental health. There is no reason to doubt the reports of people with psychosis that they received less support at school or home. Black pupils in England are more likely to be expelled than those from White UK ethnic groups, ${ }^{23}$ so this could potentially contribute to some of the excess of psychosis in Black groups. Many of the reasons given by people with psychosis for perceived discrimination are suggestive of negative self-beliefs. Such beliefs may have led people with psychosis to blame themselves for a disadvantage that might have been more accurately attributed to race, culture, class or mental illness. It has been suggested previously that negative schematic beliefs about the self might have a role in the origin and maintenance of psychosis. ${ }^{24,25}$ It is possible that correctly identifying hostility as due to another's prejudice rather than to internal factors is protective, and that those unable to do so are more vulnerable to developing psychosis.

Although our findings do not support a direct link between the perception of disadvantage and the specific emergence of persecutory delusions, perceptions of disadvantage could act synergistically with other affective mechanisms to evoke psychotic symptoms. Modern cognitive models claim that emotional processes act singly or in combination with cognitive biases in vulnerable individuals to increase the risk of positive psychotic symptom formation through the resulting appraisal patterns. ${ }^{26-29}$

Low self-esteem and poor self-concept are indicators of emotional biases that might act in such a way. The Rosenberg measure of self-esteem, sufficiently closely associated with depressed mood to serve as a proxy for affect, ${ }^{30}$ was clearly associated with psychosis in our study. Those perceiving greater disadvantage had significantly poorer self-esteem and self-concepts, although the size of the association was small. Such associations have been reported before. ${ }^{31}$ It has been suggested that extreme negative evaluations of the self and others mediate the link between emotional processes and positive symptoms of psychosis. ${ }^{24,25}$ However, this could not explain the increased rates of perception of disadvantage among Black participants because, in line with previous findings, ${ }^{32}$ ÆSOP participants from non-White ethnic groups reported higher self-esteem and self-concept scores. ${ }^{33}$ Controlling for these factors thus increased the odds ratio for the relationship between Black ethnicity and psychosis.

Our findings thus lead us to conclude that the greater disadvantages perceived by Black participants were due neither to psychotic symptoms nor to negative self-perceptions. A logical assumption would be that they were probably due to actual discriminatory experiences. Such a hypothesis should be considered with caution, as we measured perception of disadvantage rather than actual experiences. It is, however, supported by previous studies of associations of psychosis with discrimination and racist abuse. $^{3,4}$ We agree with previous authors ${ }^{5}$ that a longitudinal study - for example of people at high risk who have yet to develop psychosis - is now needed to determine whether disadvantage, perceived or actual, predicts the emergence of psychosis. Qualitative research to explore notions of perceived disadvantage across ethnic groups would also be an interesting direction for future work.

\section{Limitations of the study}

Direction of causality cannot be determined from this crosssectional study. We categorised ethnicity and religion into broad groups to ensure we had adequate power to test our hypotheses, but there might well have been diversity in the experiences of disadvantage within groups, for example the experiences of White Irish people probably differed from those of White British people. Those not interviewed because they did not speak English might have experienced particularly high levels of disadvantage. The response rate for the cases group was fairly low and this might have led to either over- or underestimation of mediation. We could not include all potential confounders; for example, we did not measure recent life events or early life experiences, which might have contributed to the participants' perceptions of disadvantage. The questions relating to perceptions of disadvantage were single-item Likert scales which had not been individually validated. We have hypothesised that the participants' perceptions of disadvantage were based on actual discriminatory experiences, but these were not measured directly.

\section{Implications of the study}

Perceived disadvantage partly mediated the relationship between psychosis and ethnicity, lending support to the hypothesis that disadvantage, perceived or actual, might contribute to the excess of psychosis observed in Black ethnic groups in the UK. If those who experience more disadvantage are indeed more prone to psychosis, it would have important consequences both for primary prevention and for treatment. However, people with psychosis are more likely to perceive that they are disadvantaged in society, even after taking into account ethnicity, social class and other socio-economic factors. Few people with psychosis attributed the disadvantage they felt to mental illness or racism, and they were more likely to attribute it to their own appearance or behaviour, or to lack of support at school or home. A longitudinal study is now needed to determine the direction of causality.

\section{Claudia Cooper, MRCPsych, MSC, PhD, Department of Mental Health Services, University College London; Craig Morgan, BA(Hons), MSC, PhD, MRC, Majella Byrne, BA, MA, MSc, PhD, DClinPsy, Cpsychol, Paola Dazzan, MSc, PhD, MRCPsych, Institute of Psychiatry, London; Kevin Morgan, PhD, Department of Psychology, University of Westminster, London; Gerard Hutchinson, MRCPsych, Psychiatry Unit, Department of Clinical Medical Sciences, University of the West Indies, Champs Fleurs, Trinidad; Gillian A. Doody, BSC, MBBS, MPhil, MD(Dist.), MRCPsych, University of Nottingham, Nottingham; Glynn Harrison, FRCPsych, Academic Unit of Psychiatry, Cotham House, Bristol; Julian Leff, FRCPsych, Department of Mental Health Sciences, University College London; Peter Jones, PhD, MRCPsych, Department of Psychiatry, University of Cambridge, Addenbrooke's Hospital, Cambridge; Khalida Ismail, MRCPsych, PhD, Robin Murray, MD, DSC, FRCP, FRCPsych, FMedSci, Institute of Psychiatry, London; Paul Bebbington, MA, MPhil, PhD, FRCP, FRCPsych, Department of Mental Health Sciences, University College London; Paul Fearon, MB, MSC, PhD, MRCPI, MRCPsych, Institute of Psychiatry, London, UK}

Correspondence: Professor Paul Bebbington, UCL Department of Mental Health Sciences, Charles Bell House, Riding House Street, London W1W 7EY, UK. Email: p.bebbington@ucl.ac.uk

First received 29 Jun 2007, final revision 5 Oct 2007, accepted 9 Nov 2007

\section{Acknowledgements}

The Aetiology and Ethnicity in Schizophrenia and Other Psychoses (ESOP) study was funded by the UK Medical Research Council. We also acknowledge the Stanley Medical Research Institute for their support. We also wish to thank the $\mathbb{A}$ ESOP researchers who helped with the data collection and the individuals who took part in the study. 


\section{References}

1 Fearon $\mathrm{P}$, Kirkbride JB, Morgan $\mathrm{C}$, Dazzan $\mathrm{P}$, Morgan $\mathrm{K}$, Lloyd $\mathrm{T}$, Hutchinson G, Tarrant J, Fung WL, Holloway J, Mallett R, Harrison G, Leff J, Jones PB, Murray RM, $\mathbb{E}$ SOP Study Group. Incidence of schizophrenia and other psychoses in ethnic minority groups: results from the MRC AESOP study. Psychol Med 2006; 36: 1541-50.

2 Sharpley M, Hutchinson G, Murray RM, McKenzie K. Understanding the excess of psychosis among the African-Caribbean population in England: review of current hypotheses. Br J Psychiatry 2001; 178, s60-8.

3 Gilvarry CM, Walsh E, Samele C, Hutchinson G, Mallett R, Rabe-Hesketh S, Fahy T, van Os J, Murray RM. Life events, ethnicity and perceptions of discrimination in patients with severe mental illness. Soc Psychiatry Psychiatr Epidemiol 1999; 34: 600-8.

4 Karlsen S, Nazroo J. The relationship between racism, social class and physical and mental health among different ethnic groups in England. Ethn Health, 2004; 9: S46-7.

5 Chakraborty A, McKenzie K. Does racial discrimination cause mental illness? Br J Psychiatry 2002; 180: 475-7.

6 Wamala S, Bostrom G, Nyqvist K. Perceived discrimination and psychological distress in Sweden. Br J Psychiatry 2007; 190: 75-6.

7 Veling W, Selten JP, Susser E, Laan W, Mackenbach JP, Hoek HW. Discrimination and the incidence of psychotic disorders among ethnic minorities in The Netherlands. Int J Epidemiol 2007; 36: 761-8.

8 World Health Organization. The ICD-10 Classification of Mental and Behavioural Disorders: Clinical Description and Diagnostic Guidelines. WHO 1992

9 Jenkins R, Meltzer $\mathrm{H}$. The National Survey of Psychiatric Morbidity in Great Britain. Soc Psychiatry Psychiatr Epidemiol 1995; 30: 1-4.

10 Bebbington PE, Nayani T. The Psychosis Screening Questionnaire. Int J Methods Psychiatr Res 1995; 5: 11-19.

11 Morgan C, Dazzan P, Morgan K, Jones P, Harrison G, Leff J, Murray R, Fearon $P$, ÆESOP Study Group. First episode psychosis and ethnicity: initial findings from the AESOP study. World Psychiatry 2006; 5: 40-6.

12 Kirkbride JB, Fearon P, Morgan C, Dazzan P, Morgan K, Tarrant J, Lloyd T, Holloway J, Hutchinson G, Leff JP, Mallett RM, Harrison GL, Murray RM, Jones PB. Heterogeneity in incidence rates of schizophrenia and other psychotic syndromes: findings from the 3-center $\mathbb{F}$ ESOP study. Arch Gen Psychiatry 2006; 63: 250-8.

13 Mallett R. Sociodemographic Schedule. Institute of Psychiatry, 1997.

14 Bhugra D, Bhui K, Mallett R, Desai M, Singh J, Leff J. Cultural identity and its measurement: a questionnaire for Asians. Int Rev Psychiatry 1999; 11: 244-9.

15 World Health Organization. Schedules for Clinical Assessment in Neuropsychiatry (SCAN) Version 2.0. WHO, 1994.

16 Wing JK, Sturt E. The PSE-ID-CATEGO System: Supplementary Manual. Medical Research Council, 1978.
17 Roe D. A prospective study on the relationship between self-esteem and functioning during the first year after being hospitalized for psychosis. $J$ Nerv Ment Dis 2003; 191: 45-9.

18 Rosenberg M. Society and the Adolescent Self-image. Princeton Associated Press, 1965.

19 Robson P. Development of a new self-report questionnaire to measure selfesteem. Psychol Med 1989; 19: 513-18.

20 Baron RM, Kenny DA. The moderator mediator variable distinction in social psychological research - conceptual, strategic, and statistical considerations. J Pers Soc Psychol 1986; 51: 1173-82.

21 Raune $D$, Bebbington $P$, Dunn GD. Event attributes and the content of psychotic experiences in first episode psychosis. Psychol Med 2006; 36 221-30

22 Garety PA, Bebbington P, Fowler D, Freeman D, Kuipers E. Implications for neurobiological research of cognitive models of psychosis. Psychol Med 2007; 37: 1377-91.

23 British Broadcasting Corporation News. Q\&A: Expelled Pupils. BBC, 2004 (http://news.bbc.co.uk/1/hi/education/4022123.stm).

24 Fowler D, Freeman D, Smith $B$, Kuipers $E$, Bebbington $P$, Bashforth $H$, Coker S, Hodgekins J, Gracie A, Dunn G, Garety P. The Brief Core Schema Scales (BCSS): psychometric properties and associations with paranoia and grandiosity in non-clinical and psychosis samples. Psychol Med 2006; 36 749-59.

25 Smith B, Fowler DG, Freeman D, Bebbington P, Bashforth H, Garety P, Dunn G, Kuipers E. Emotion and psychosis: links between depression, self-esteem, negative schematic beliefs and delusions and hallucinations. Schizophr Res 2006; 86: 181-8.

26 Garety $P$, Kuipers E, Fowler D, Freeman D, Bebbington PE. A cognitive model of the positive symptoms of psychosis. Psychol Med 2001; 31: 189-95.

27 Morrison AP. The interpretation of intrusions in psychosis: an integrative cognitive approach to hallucinations and delusions. Behav Cogn Psychother 2001; 2: 257-76.

28 Freeman $D$, Garety $P$, Kuipers E, Fowler D, Bebbington PE. A cognitive model of persecutory delusions. Br J Clin Psychol 2002; 41: 331-47.

29 Birchwood M. Pathways to emotional dysfunction in first-episode psychosis. Br J Psychiatry 2003; 182: 373-5.

30 Aluja A, Rolland J-P, Garcia LF, Rossier J. Dimensionality of the Rosenberg Self-Esteem Scale and its relationships with the three-and the five-factor personality models. J Pers Assess 2007; 88: 246-9.

31 Barrowclough C, Tarrier N, Humphreys L, Ward J, Gregg L, Andrews B. Selfesteem in schizophrenia: relationships between self-evaluation, family attitudes, and symptomatology. J Abnorm Psychol 2003; 112: 92-9.

32 Sharpley MS, Peters E. Ethnicity, class and schizotypy. Soc Psychiatry Psychiatr Epidemiol 1999; 34: 507-12.

33 Byrne M. A baseline and 8-year follow-up study of first onset psychosis: selfesteem and locus of control in the Aetiology and Ethnicity of Schizophrenia and Other Psychosis (AESOP) Study. DClinPsy Thesis, King's College London, 2006. 